\title{
Gianmario Borio
}

\section{Das Musikinstitut der Fondazione Giorgio Cini}

\author{
Quellenbasierte Forschung im gegenwärtigen Horizont
}

\begin{abstract}
In 2020, the Institute of Music of the Fondazione Giorgio Cini celebrated its 35th birthday. Its archive preserves 26 collections belonging to Italian composers and musicians of the 20th century: a significant quantity of sources encompassing several generations and aesthetic orientations (from Casella to Romitelli, from Rota to Manzoni etc.). The state of current discourse and new technologies are leading to a re-orientation of archival work. First, it can contribute to a more detailed picture of musical facts and personal interactions. Second, digital technologies create the conditions for new ways of evaluating the data found in different sources; and third, an archive is now increasingly conceived as a service and the use of its resources is not limited to historiographical research but also encompasses the dissemination of knowledge and musical practice. The book series „The Composer's Workshop”, the online-journal „Archival Notes” and the workshop cycle „Research-led Performance” are examples of the wide range of activities connected with our archive.
\end{abstract}

Als 1985 das Musikinstitut (Istituto per la Musica) der Fondazione Giorgio Cini in Venedig eingeweiht und Giovanni Morelli zum Direktor ernannt wurde, umfasste sein Archiv vor allem Bestände des sonst verstreuten Nachlasses von Ottorino Respighi sowie die Sammlung von Gian Francesco Malipiero, der eine enge Freundschaft mit Vittore Branca, dem Generalsekretär der Stiftung, gepflegt hatte. Malipiero wurde in die Tätigkeiten der Stiftung bereits 1956 einbezogen, als das Centro di Cultura e Civiltà entstand, das Theater und Musik besondere Aufmerksamkeit schenkte; daraufhin initiierte der Komponist die kritische Ausgabe der Werke von Giovanni Gabrieli und eine Reihe von Studien zur Renaissance-Musik in Venedig. ${ }^{1}$ Der Schwerpunkt des musikalischen Sektors lag damals auf der photographischen Reproduktion von Quellen aus der Renaissance und dem 17. Jahrhundert sowie auf der Sammlung Ulderico Rolandi, die aus einer beträchtlichen Anzahl von Opernlibretti aus verschiedenen Epochen besteht. In diesem Rahmen stellten die Personensammlungen Respighi und Casella eher eine Ausnahme dar. Die vorwiegende Orientierung war, das

1 Eine Tagung 1972 wurde Malipieros Werk und Denken gewidmet. Das daraus entstandene Buch gehört zu den ersten musikwissenschaftlichen Veröffentlichungen der Fondazione; vgl. Mario Messinis (Hg.), Omaggio a Malipiero, Firenze 1977 (Fondazione Giorgio Cini. Studi di musica veneta 31).

Kontakt: Gianmario Borio, gianmario.borio@unipv.it 
Atelier eines Komponisten mit seinen Objekten in ihrer ursprünglichen Disposition abzubilden, was bei der Einrichtung des Respighi-Saals noch heute zu beobachten ist. Die Rahmenbedingungen des Schaffens und die Aura der im Raum aufgestellten Gegenstände bildeten den Mittelpunkt des Interesses. Die Bewahrung des historischen Gedächtnisses, die bald zum Hauptkriterium für die Gründung und Pflege von Archiven hier und anderweitig avancierte, hatte eine sekundäre Bedeutung; denn die wissenschaftliche Ausarbeitung der Quellen wurde als ein Geschäft für einen kleinen Kreis von Fachleuten angesehen.

Eine Wende leitete Morelli im ersten Jahrzehnt des Institutsbestehens ein. ${ }^{2}$ Die Erwerbung der Nachlässe von Alfredo Casella (1992), Nino Rota (1995) und Camillo Togni (2000) bestimmte eine Differenzierung, die sich in der archivalischen Arbeit und der Konzeption der Forschungsprojekte niederschlug. Erstens entstand ein Interesse an mühelos nachschlagbaren Inventaren; zweitens galt es, Projekte zu entwickeln, um mit Hilfe der aufbewahrten Quellen Lücken in der musikhistorischen Forschung zu schließen. Dies betraf vor allem „primäre“ Materialien: quellengeprüfte Werkverzeichnisse sowie Veröffentlichungen von Schriften der Komponisten und ihrer Briefwechsel. ${ }^{3}$ Solche Projekte waren monographisch angelegt: im Mittelpunkt standen der Komponist, sein Leben, seine Überzeugungen, seine Beziehungen zu Personen und Institutionen. Morelli war aber zugleich auch an der Außenperspektive interessiert, weswegen er das 1993 gegründete, in unmittelbarer Nachbarschaft gelegene Archivio Luigi Nono in das Forschungsprogramm des Musikinstituts einbezog. ${ }^{4}$

Als ich 2012 die Leitung des Musikinstituts übernahm, hatte sich die archivalische Landschaft tiefgreifend verändert. Bedeutende Archive zur Musik des 20. Jahrhunderts wie die Paul Sacher Stiftung in Basel und das Musikarchiv der Berliner Akademie der

2 Vgl. Giovanni Morelli, I Fondi inerenti la musica: Malipiero, Boito, Casella, Milloss, Rota, Rudge, Sartori, Togni, in: Ulrico Agnati (Hg.), La Fondazione Giorgio Cini. Cinquant’anni di storia, Milano 2001, S. 125-134. Vgl. auch Morellis Vortrag auf der Tagung Conservare il Novecento (Ferrara 2000), nachschlagbar in http://www.aib.it/aib/commiss/cnsbnt/morelli.htm. Wenn nicht anders angegeben, sind alle Internetressourcen letztmalig am 20.9.2020 konsultiert worden.

3 Vgl. Roberto Calabretto (Hg.), Alfredo Casella. Gli anni di Parigi. Dai documenti, Firenze 1997 (Fondazione Giorgio Cini. Studi di musica veneta 25); Cecilia Gibellini (Hg.), Carteggi e scritti di Camillo Togni sul Novecento internazionale, Firenze 2006 (Fondazione Giorgio Cini. Studi di musica veneta. Archivio Camillo Togni 3).

4 Vgl. die beiden Forschungsberichte Gianmario Borio/Giovanni Morelli/Veniero Rizzardi (Hg.), La nuova ricerca sull'opera di Luigi Nono, Firenze 1999 (Fondazione Giorgio Cini. Studi di musica veneta. Archivio Luigi Nono. Studi 1); dies. (Hg.), Le musiche degli anni Cinquanta, Firenze 2004 (Fondazione Giorgio Cini. Studi di musica veneta. Archivio Luigi Nono. Studi 2); sowie die Briefwechselausgaben Antonio Tru du (Hg.), Luigi Nono. Carteggi concernenti politica, cultura e Partito Comunista Italiano, Firenze 2008 (Fondazione Giorgio Cini. Studi di musica veneta. Archivio Luigi Nono. Studi 3); Angela Ida De Benedictis/Ulrich Mosch (Hg.), Alla ricerca di luce e chiarezza. L'epistolario Helmut Lachenmann - Luigi Nono (1957-1990), Firenze 2012 (Fondazione Giorgio Cini. Studi di musica veneta. Archivio Luigi Nono. Studi 4). 
Künste hatten neue Standards der Quellenforschung etabliert. Studien zu den Zeugnissen der kompositorischen Arbeit - vor allem Skizzen und Entwürfe - haben über die Konzeption einzelner Werke, ihre Grammatik und ihre theoretischen Hintergründe wertvolle Auskunft gegeben. Die Musikgeschichte des 20. Jahrhunderts begann ein differenzierteres Bild als früher zu zeigen. Die Komplexität und bisweilen auch Widersprüchlichkeit individueller Laufbahnen; die Überschneidungen von Kompositionstechniken und ästhetischen Vorstellungen; die gemeinsame Basis der theoretischen Fragen, an denen sich die kompositorische Arbeit orientiert; die Bildung von Interessengruppen bzw. einflussreichen Cliquen - all das wurde nun durch Archivarbeit greifbar und sollte es auch im Musikinstitut der Fondazione Giorgio Cini werden.

Die Erforschung der archivalischen Materialien ist heute somit zur unabdingbaren Etappe der historiographischen Arbeit geworden. Ihr Nutzen wird inzwischen auch von der Musiktheorie und der Musikästhetik erkannt, und neuerdings zeigt auch die Musikethnologie Interesse an der Bewahrung des archivalischen Erbes. ${ }^{5}$ Ihrerseits hat die Musikphilologie die aus den Studien des Schaffensprozesses gewonnenen Ergebnisse positiv aufgenommen: das Autograph, das den „Autorenwillen“ dokumentiert, und die Abschrift, die ein Stadium in der „Textgeschichte“ belegt, sind nicht mehr die einzigen Forschungsobjekte, sondern es werden auch andere Quellentypen herangezogen. Die Archive zur Musik des 20. Jahrhunderts zeigen die Vielfalt der Quellen, die einer allmählich mehrschichtig verstandenen Forschung dienlich sein können. Entwürfe, Niederschriften und Revisionen verbinden sich mit einem mannigfaltigen Repertoire von Skizzen: Bloc-notes, Hefte, Tabellen, Zeichnungen usf. Dazu können ferner Bearbeitungen literarischer Texte für Vokalwerke hinzukommen, Projekte zur Inszenierung von Bühnenwerken, präskriptive oder deskriptive Partituren für elektronische Musik, Synchronisierungspläne für Film und Fernsehen, Materialien zur Vermittlung im Unterricht oder in den Medien, Niederschriften von Aufsätzen, Aufzeichnungen in Büchern oder Partituren anderer Autoren, Briefwechsel, Tagebücher, Foto- und Video-Aufnahmen, Tonaufzeichnungen von Proben und Aufführungen. Potentiell kommuniziert jede Quelle mit den anderen; je nach Fragestellung können sich Netzwerke und Hierarchien unterschiedlicher Materialien formieren.

Die Bestände des Musikarchivs der Fondazione Giorgio Cini haben sich inzwischen nahezu verdoppelt. Die Einbindung der Nachlässe von Niccolò Castiglioni, Renato De Grandis, Domenico Guaccero, Egisto Macchi, Franco Oppo, Fausto Romitelli, Roman Vlad und anderer wie auch die der Sammlung Giacomo Manzoni haben einen Aspekt des Archivs hervorgehoben: dass es selbst den Charakter eines Netzwerks hat. Die italienische Musikgeschichte des 20. Jahrhunderts zeigt sich hier als ein

5 Aus diesem gegenseitigen Interesse hat sich in diesen Jahren die Mitarbeit mit dem von Giovanni Giuriati geleiteten Istituto Interculturale di Studi Musicali Comparati der Fondazione Giorgio Cini verstärkt. Darin sind Sammlungen zur Tätigkeit von Alain Danielou sowie zur Weltmusik aufbewahrt. Vgl. https://archivi.cini.it/musicacomparata/home.html. 
Komplex von Werken, Ideen und Beziehungen, deren Dynamik sich sowohl synchron als auch diachron entfaltet. Die Bearbeitung und Veröffentlichung der Metadaten, die dank des technologischen Fortschritts und der finanziellen Unterstützung durch die Fondazione Giorgio Cini möglich wurden, macht dies besonders deutlich. ${ }^{6}$ Das Profil der heutigen Forschung spiegelt die Vernetzung von Personen und Institutionen wider, die das 20. Jahrhundert insgesamt gekennzeichnet hat. Analog zur Kommunikation einzelner Sammlungen miteinander zeigt auch das Archiv eine zentrifugale Tendenz: es verlangt nach Verbindungen zu anderen Archiven.

Die Vernetzung von Personen und Institutionen wird bei den Festivalprogrammen besonders greifbar. Die bisherigen Berichte über Festtage und Konzertreihen zielen meistens auf die Darstellung der Ereignisse, wie sie tatsächlich geschehen sind und rezipiert wurden; selten kommt eine Tiefenperspektive ins Spiel, die die Zusammensetzung von Programmen und Interpreten in Bezug auf analoge Initiativen zu erklären vermag. Eine vergleichende Studie der Konzertprogramme von Le Domaine Musical, der Donaueschinger Musiktage, des Warschauer Herbstes, der Reihe „Incontri musicali“ und der Darmstädter Ferienkurse könnte sowohl identische Überzeugungen als auch spezifische Züge der einzelnen Institutionen beleuchten. Die Schaffung einer europäischen Plattform, die Quellen dieses Typus zusammenstellt, wäre für die Forschung von beträchtlichem Nutzen. Derartigen Untersuchungen kommt die gegenwärtige Technologie besonders entgegen. Die Systeme OCR (Optical Character Recognition) und ICR (Intelligent Character Recognition) erlauben eine Befragung von Daten, deren Genauigkeit und Geschwindigkeit alle vorangegangenen Verfahren überschreitet. Sie können bei einem Corpus besonders wirkungsvoll sein, das aus unterschiedlichen Quellentypen (teils gedruckt, teils handgeschrieben) besteht. Anders als in den alten Verzeichnissen auf Papier bieten diese Systeme keine vorbestimmte Sequenz von Daten an; die Suche orientiert sich vielmehr an Typ und Inhalt der Fragen, die die Forschenden stellen. Ein weiterer Schritt ist durch das Natural language processing gegeben, ein technologisches Verfahren, das die Inhalte einer - wenn auch disparaten - Reihe von Dokumenten kategorial deuten kann, d.h. mögliche Wege aufzeigt, um die in den Quellen enthaltene Information dem ursprünglichen Kontext bzw. einem spezifischen Bereich des Forschungsprojekts zuzuordnen. Die Digitalisierungskampagne des Musikinstituts ist unter diesen Voraussetzungen in das umfangreichere Projekt „ARCHiVe“ eingegliedert, das das Gesamtreservoir der in der Fondazione Giorgio Cini aufbewahrten historischen Bestände umfasst. ${ }^{7}$

Im Musikinstitut werden die Katalogisierung und Bewahrung der Quellen von Eingriffen $\mathrm{zu}$ ihrer wissenschaftlichen Verwertung flankiert. Es leistet finanzielle Unterstützung für individuelle Forschungsvorhaben und Studiengruppen, die sich

6 Vgl. https://archivi.cini.it/istitutomusica/home.html.

7 https://www.cini.it/en/institutes-and-centres/archive-analysis-and-recording-of-cultural-heritagein-venice. 
besonders mit quer durch die Sammlungen gehenden Fragen beschäftigen. Die 2016 gegründete Zeitschrift „Archival Notes“ berücksichtigt die komplexen Wege des Schaffensprozesses und die Interaktionen, welche die Musikkultur des 20. Jahrhunderts geprägt haben. ${ }^{8}$ Sie wurde als Forum unterschiedlicher Erfahrungen der Geschichtsschreibung und als Plattform zum Informationsaustausch zwischen verwandten Institutionen konzipiert. Der erste Band, über Briefwechsel, ist für diese Ausrichtung nicht weniger repräsentativ als der Fokus auf die online-Datenbank des Internationalen Musikinstituts Darmstadt in Band 3 oder der auf die Erforschung der Quellen zur Filmmusik im kommenden Band 5. Ein weiterer Bereich, auf den „Archival Notes“ mit erhöhter Aufmerksamkeit blickt, ist die Untersuchung des Verhältnisses von musikalischem Leben und politischen Strukturen. In den bisherigen Bänden sind zwei Aufsätze erschienen, die auf Malipieros Rezeption in Nazi-Deutschland und seine Stellungnahme für den Comitato Nazionale di Liberazione eingehen, der ihn gleich nach Kriegsende als Komplizen der faschistischen Regime anklagte. ${ }^{9}$ Eine präzise Darstellung von Ereignissen und Verhaltensweisen wäre ohne den Bezug auf die archivalischen Quellen nicht möglich. Die Arbeit im Archiv erlaubt es, nicht nur eine realitätsgerechte Darstellung anzubieten, sondern auch den Abstand zwischen öffentlicher Stellungnahme und privater Gesinnung zu ermessen. In diesem Bereich gibt es noch relevante Fragen, auf die das Beziehungsgeflecht unterschiedlicher Quellen Auskunft geben könnte: das Entstehen eines musikalischen Nationalismus, der sich um den ersten Weltkrieg zum politischen gesellte; die Reaktion der Komponisten auf die gedrückte Stimmung der Kriegszeit; der Einfluss der Kulturpolitik der Kommunistischen Partei bis zur „Biennale del dissenso“ von 1977.

Die Potentiale der heutigen Medienlandschaft und das Aufblühen eines partizipatorischen Begriffs von Demokratie konfrontieren ein Archiv mit neuen Herausforderungen. ${ }^{10}$ Unter den gegenwärtigen Bedingungen kann sich der Existenzgrund eines Archivs nicht in sich selbst - als Ort der Bewahrung des kollektiven Gedächtnisses - erschöpfen; seine Wirkung auf die Gesellschaft muss auch berücksichtigt werden. Einen unmittelbaren Niederschlag im konkreten Musikleben kann die Veröffentlichung verschollener oder schwer zugänglicher Werke bewirken. Zu Beginn des 20. Jahrhunderts hat das Musikinstitut dies durch die Bekanntmachung der Musik, die Nino Rota außerhalb des Filmbetriebs komponiert hat, mit der Unterstützung des Schott-Verlages verfolgt; dies führte zur Entdeckung bedeutender Werke, zum Beispiel

8 http://onlinepublishing.cini.it/index.php/arno/index.

9 Tobias Reichard, ,Malipiero Germanised“ - Traces of Cultural Usurpation in Nazi Germany, in: Archival Notes 2 (2017): http://onlinepublishing.cini.it/index.php/arno/article/view/60/186; und Paola Cossu, Gian Francesco Malipiero, Esalazioni epurative, in: Archival Notes 4 (2019): http:// onlinepublishing.cini.it/index.php/arno/article/view/96.

10 Vgl. dazu Gianmario Bori o, Music Archives in the Twenty-First Century: The Challenges of Politics and Technology, in: Archival Notes 3 (2018), pp. 137-145: http://onlinepublishing.cini.it/index.php/ arno/article/view/106/212. 
seiner Ersten und Zweiten Symphonie oder des Bühnenwerks „Aladino e la lampada magica“. In diesen Bereich gehört auch die Vervollständigung und Veröffentlichung des Klavierkonzerts, an dem Camillo Togni in seinen letzten Lebensjahren arbeitete. ${ }^{11}$ Mit der Erwerbung des Nachlasses Castiglioni ist eine beträchtliche Anzahl von Autographen aus unterschiedlichen Schaffensperioden aufgetaucht; demzufolge wurde ein wissenschaftlicher Beirat gebildet, der ein editorisches Programm zur Veröffentlichung dieser bisher unbekannten Werke dank des Einsatzes des Verlags Suvini-Zerboni beschlossen hat.

Eine noch engere Beziehung zur Aufführungspraxis wurde durch die WorkshopReihe „Research-led Performance“ anvisiert, die 2016 anlässlich eines gemeinsamen Projekts mit dem Orpheus Instituut von Gent entstand. Der Titel bezieht sich auf die gegenseitige Integration von Disziplinen und Kompetenzen. Die Reihe gründet auf einer doppelten Prämisse: dass einerseits die Aufführungspraxis durch die Ergebnisse der Quellenforschung und der theoretischen Durchdringung der Werke auf eine solidere Grundlage gestellt werden kann, und andererseits dass die musikwissenschaftliche Arbeit von den Erfahrungen des Spielens und Hörens wesentlich profitieren kann. Eine im 20. Jahrhundert weit verbreitete Überzeugung, die auf die Aussagen der beiden Gründungsväter der Neuen Musik, Schönberg und Strawinsky, zurückgreifen konnte, besagt, dass die Komponisten der post-tonalen Musik ihren Sinn für die Mittel und Funktionen der Notenschrift soweit entwickelt hatten, dass ihre Intention von der Partitur vollständig vertreten wurde; um dem Werkinhalt gerecht zu werden, bräuchten nämlich die Musiker bloß die Zeichen in Klänge zu übertragen. Diese Überzeugung hatte eine historische Rechtfertigung: es galt, standardisierten Ausdrucksformen sowie einer in der tonalen Musik verankerten Rhetorik vorzubeugen; die „notengetreue“ Wiedergabe des Notentextes war außerdem gewissermaßen eine Notwendigkeit, da sich die musikalische Sprache so gewandelt hatte, dass eine vom Text abweichende Aufführung schnell in Unverständlichkeit münden konnte. Der subjektive Anteil der Interpretin bzw. des Interpreten rückte aber damit in den Hintergrund und wurde manchmal sogar unterdrückt. Auf einer solchen ideologischen Basis ist eine Auseinandersetzung mit auktorialen Quellen kaum denkbar. Die Situation hat sich inzwischen grundsätzlich verändert, nicht zuletzt wegen eines weit entwickelten Bewusstseins für die historische Verortung der zu spielenden Werke durch ihre Interpreten. In den Veranstaltungen von „Research-led Performance“ geht es um die Verbreitung historischen Wissens in Bereichen, die von sich aus auf das technische Können und seine Verbesserung konzentriert sind. Die Quellen kommen wortwörtlich „zum Erklingen“; sie nehmen eine akustische Körperlichkeit auf, die die in der Erforschung „visueller“ Quellen herangewachsenen Kenntnisse ergänzt und unterstützt.

11 Paulo De Assis (Hg.), Domani l'aurora. Ripristino ricostruttivo del „Concerto per pianoforte e orchestra“ incompiuto (1993) di Camillo Togni, Firenze 2004 (Fondazione Giorgio Cini. Studi di musica veneta. Archivio Camillo Togni 2). 
Heute befinden sich die Archive zur Musik des 20. Jahrhunderts an einer historischen Schwelle. Chronologisch ist diese durch das 21. Jahrhundert festgelegt, das den Horizont setzt, in dem sich die gegenwärtigen Forschungen in den Bereichen Musikgeschichte und -wissenschaft abspielen; technologisch fällt sie mit dem Einsatz der digitalen Klangforschung und -bearbeitung durch die Komponisten selbst zusammen. In der Sammlung Fausto Romitelli befindet sich zum Beispiel ein MacIntosh Computer des Typus LC 475, Baujahr 1993. Jüngst hat Alessandro Olto, ein Stipendiat des Musikinstituts, die library rekonstruiert, die der Komponist vor allem während seiner Mitarbeit am Pariser IRCAM (1993-1996) in LISP-Codex verfasst hatte, und sie dann in das Umfeld vom OpenMusic-Software übertragen. Die Forschung muss also auf technisches Können zurückgreifen, um die vom Patchwork generierten Dokumente herauszufiltern; daraufhin wird ein Dossier geschaffen, das sowohl den Kodex in textlicher Form als auch seine graphische Visualisierung enthält. Im Fall Romitellis überliefern die Patch das harmonische Grundmaterial für mehrere Kompositionen, was den Quellenstand wesentlich bereichert. Sie enthalten aber auch graphische Darstellungen von generischen Funktionen, die als ein Stück impliziter Musiktheorie mit Romitellis theoretischen Schriften kommunizieren. Die Gegenüberstellung dieser Befunde mit den schriftlichen Quellen zum Schaffensprozess trägt dazu bei, die Kenntnisse über einen zentralen Aspekt seiner Kompositionstechnik zu verbessern: die Suche nach Beziehungen zwischen den spektralen Manipulationen (Klangverzerrungen, FM-Verfahren usw.) und einer Verarbeitung der Intervalle, die sich im Bereich der seriellen und postseriellen Musik entwickelt hat.

In den Archiven der Musik des 20. Jahrhunderts überschneiden sich notwendigerweise die Epochen - eine Grenzüberschreitung, die durch den Wechsel der Quellentypen und ihrer Hierarchie ersichtlich wird. Die musikalische Ausbildung und der kulturelle Hintergrund lebender Komponistinnen und Komponisten liegen im 20. Jahrhundert; sie operieren aber heute in einer gänzlich veränderten Situation. Der Wandel betrifft sowohl die soziale als auch die technologische Ebene - oder besser: sie manifestiert sich in einer Verquickung dieser beiden Ebenen. Die digitalen Technologien haben neue Vorstellungen der musikalischen Schrift eingeführt. Der Computer ist nicht nur eine Verlängerung der überlieferten Techniken musikalischen Notierens, sondern greift in die Dynamik des kompositorischen Schaffens direkt ein, da er automatische Verfahrensweisen zur Fertigung von Klangstrukturen anbietet. Die Algorithmen erlauben eine sofortige Überprüfung aller Dimensionen des akustischen Phänomens, setzen also die Bedingungen zu einem permanenten Feedback zwischen Komponist und Klang, Vorstellung und Realisierung. Die Stadien eines solchen graduellen Fokussierens auf das erwünschte Ergebnis sind in den gespeicherten Dateien dokumentiert; jedoch verlangt die Interpretation dieser neuen Quellen auch neue Kompetenzen seitens der Musikforscherinnen und Musikforscher.

Der Einbezug der digitalen Technologien betrifft aber nicht nur den Bereich, der Computer assisted composition genannt wird, sondern umfasst auch Aspekte des Performativen, die dadurch das Quellenreservoir zur kompositorischen Arbeit berei- 
chern. Die Live-Elektronik etwa führt Variabilität in der Aufführung eines Werkes ein und schafft damit ein flexibles Feld seiner Daseinsformen, das durch die Notenschrift schwer darstellbar ist. Damit wird der normative Charakter des Notentextes von den Komponisten selbst, also in einer Innenbewegung der Geschichte kompositorischer Technik, in Frage gestellt, noch bevor die Theoretiker der Performance studies ihre Herausforderungen lancierten. Die unter der Leitung von Morelli im Jahr 2007 veranstalteten Seminare zur Aufführungspraxis von Nonos Spätwerken können für diese Problematik als bezeichnend gelten. ${ }^{12}$ Schließlich hat sich das Interesse der Komponisten für die audiovisuellen Gebilde über die traditionelle Filmmusik hinaus entwickelt, was in Romitellis „Index of Metals“ besonders deutlich wird. Auch in diesem Bereich finden wir eine Verbindung der traditionellen Mittel zur Festlegung musikalischer Gedanken mit technologischen Verfahrensweisen, deren Quellen nur auf elektronischen Trägern zugänglich sind.

12 Vgl. https://www.cini.it/eventi/corso-dinterpretazione-sulle-opere-di-luigi-nono-con-live-electro nics-e-con-nastro-magnetico-it. 